\title{
Scenario-Driven Search for Pedestrians aimed at Triggering Non-Reversible Systems
}

\author{
Alberto Broggi, Pietro Cerri, Luca Gatti, Paolo Grisleri \\ VisLab - Dipartimento di Ingegneria dell'Informazione \\ Università degli Studi di Parma, ITALY \\ www.vislab.it \\ \{broggi, cerri, lucag, grisleri\}evislab.it
}

\author{
Ho Gi Jung, JunHee Lee \\ Central R\&D Center \\ MANDO Corporation \\ Yongin-Si 446-901, Korea \\ \{hgjung, leejunhee\}@mando.com
}

\begin{abstract}
This paper presents the results of an innovative approach to pedestrian detection for automotive applications in which a non-reversible system is used; therefore the aim is to reach a very low false detection rate, ideally zero, by searching for pedestrians in specific areas only.

The great advantages of such an approach are that pedestrian recognition is performed on limited image areas -therefore boosting its timing performance- and no assessment on the danger level is finally required before providing the result to either the driver or an on-board computer for automatic manoeuvres.

This system has been extensively tested on two prototype vehicles equipped with one laserscanner, one camera, and brakeby-wire technology both in Italy and Korea; this paper describes the extensive tests and shows performance measurements.
\end{abstract}

\section{INTRODUCTION}

As presented in an earlier paper [1], traditional approaches for the detection of pedestrians in front of a moving vehicle may suffer from a high number of false detections. These approaches are based on the full search for pedestrians in the whole area in front of the vehicle. Potential candidates are located using pedestrian characteristics [2] such as shape, symmetry, texture, motion, periodicity of human legs motion. When fusion between different sensing technologies is used [3] [4], being it high-level or low-level, each sensor searches for pedestrian-specific features.

As for many Advanced Driving Assistance Systems, perception is just one of the many steps: it is generally followed by a decision/planning step, and finally -in case the system needs to automatically trigger specific maneuvers- by actuation. Indeed every single step must be as robust as possible and feed reliable results to the next step, however, when the final step is a non-reversible action (such as an automatic maneuver) then perception really needs to be error-free. In this case, in fact, the complete system must be reliable enough to have an extremely low probability to trigger the non-reversible action when it is not required since such invasive actions might become a threat to other road participants.

In the case of pedestrian detection systems, actuation might be as light as an audible warning to the driver, or as invasive as an automatic braking just before hitting the pedestrian. Indeed driver warning is classified as a reversible action, although a large number of false warnings (generated by false positives in the detection step) might irritate the driver, leading to lowering the confidence in its performance. Automatic braking is defined to be a non-reversible action, whose activation must be carefully evaluated to avoid possibly worse consequences in case of false alarms. The same holds for other non-reversible systems, such as the firing of collision mitigation systems like smart airbags, or the execution of automatic pedestrian avoidance maneuvers.

As a clear consequence, when the final goal of an Advanced Driving Assistance System is the firing of non-reversible systems, the detection step must deliver almost perfect performance. In traditional approaches, as mentioned above, the localization of dangerous pedestrians follows a two-step process (see figure 1):

- first all pedestrians are detected in front of the vehicle;

- then the list of pedestrians is scanned to filter out the ones that do not represent possible threats, thus keeping the dangerous pedestrians only.

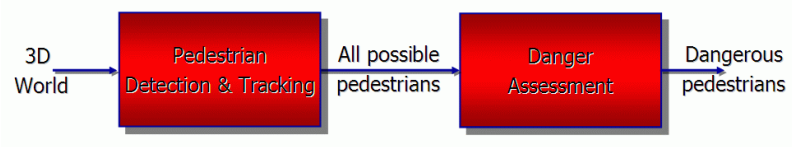

Fig. 1. The traditional two-step process for pedestrian detection: the mere detection and tracking is followed by the assessment on the danger level which acts as a filter. The result is the list of dangerous pedestrians, if any.

The first step may incorporate feature-based detection, tracking, learning, and other complex processings linked to the pedestrian's appearance; the second step needs to assess the position and direction of the pedestrian [5] with respect to vehicle motion, the probability for the pedestrian to change direction and velocity, and other environmental and behavioral characteristics that are intrinsically tied to the pedestrian nature and the environment in which the pedestrian itself is moving.

Both of these two steps must be successful in order to hold correct results at the end. Unfortunately, the literature shows that current performance of the first step are very far from ideal (and the same, and even worse numbers, apply to the second step): currently, a value of 0.01 false detections per frame is considered a good result concerning the ability to recognize the presence of a pedestrian. However, time-wise, when the system works at $10 \mathrm{~Hz}$ (10 frames per second), a false alarm is generated every 10 seconds. This is clearly unacceptable for systems whose target is the firing of nonreversible systems: we are still orders of magnitude away from 
the perfect pedestrian detector.

Anyway, while someone could theoretically design the first step to work perfectly in every situation, environment, time of day, temperature, and season, and being able to detect every single pedestrian -even partly hidden ones, - the second step, on the other hand, hides behavioral attitudes which are extremely improbable -if not impossible- to be classified, decoded, and therefore anticipated: although it can be theoretically possible to detect a pedestrian standing on the road edge, it would be quite challenging to assess the probability that the pedestrian starts walking and crosses the road while our vehicle is approaching.

Given the above considerations on the current low state-ofthe-art detection performance and the weak plausibility of the realization of a working pedestrian behavioral model, it would be interesting to consider whether continuing with this twostep approach might really lead to a working system in the near future. When checking the curve plotting the advances in terms of overall performance against time, do we still see space for improvement or have we already passed the saturation knee?

VisLab, besides following this traditional method, started a new approach and put more energy into the problem to help escaping from the local minimum that seems to be trapping most research projects. The new approach, called SDS (Scenario-Driven Search), was designed to solve the problem of pedestrian detection in specific scenarios: instead of trying to develop a system that is able to handle every situation, the system developed by VisLab, in cooperation with Mando, recognizes specific environments and performs a specific search corresponding to the given scenario. In particular, the system described in this paper was designed to work in a particularly challenging urban scenario, in which traditional pedestrian detection approaches would yield nonoptimal results.

The scenarios that are considered here refer to the most common urban situations, in which the presence of a pedestrian poses a serious problem that could be mitigated by an early detection. In particular, when vehicles are moving on an urban road, the most common threat that a pedestrian may pose -therefore requiring a successful detection- is road crossing. Stopped vehicles on the road or on the road edge create a completely new set of scenarios, in which the presence of a pedestrian might be definitely dangerous.

The underlying idea of our SDS applied to this specific scenario is to localize stopped vehicles and then search for pedestrians in their close proximity or in the areas partly hidden by them. These stopped vehicles, whose edges will trigger the search for pedestrians, may be parked cars on the road edge, vehicles temporarily stopped on the road, or vehicles queued in a line in front of a traffic light, zebra crossing, or simply jammed.

Figure 2 shows some examples of situations in which the visibility of a crossing pedestrian is partly or completely occluded by stopped vehicles, and highlights, for each situation, the areas on which the system will perform a check for the presence of a possible pedestrian.
In other words, this paper focuses on the detection of pedestrians appearing just behind non-moving infrastructures like stopped vehicles; pedestrians that are clearly visible in the frontal area of the vehicle are also detected, but they are treated differently, as it will be discussed in Section IV.

The idea of focussing on a specific scene or scenario (referring to a dynamic or a static environment, respectively) is not new to pedestrian detection systems: in 2002 Franke and Heinrich [6] developed a module able to detect balls (which are usually a strong signature of the presence of a child). Another example of very specific systems is the one developed by Curio et al. [7] which was based on the visual localization of the specific moving pattern of human legs.

This project is a cooperation between VisLab (Italy) and Mando (South Korea) and is aimed at producing a system able to detect possible threats and brake in case the danger level is not reduced by warning the driver and the pedestrian about the possible impact. Zero false alarms is the final goal of the detection system: Section V presents the results on an exhaustive set of situations which demonstrates that the idea of following an approach different form the traditional one truly payed off.

\section{The Test Vehicle}

This section describes the perception system installed onboard our test vehicle as well as sensors position and capabilities.

A Hyundai Grandeur has been modified in order to accommodate sensors and processing unit keeping in mind to reduce the impact on the external look of the car.

An AVT Guppy F-036B camera, has been placed on the right side of the internal rearview mirror. Its sensor produces grayscale images with a geometry of $752 \times 480$ pixels. The aspect ratio, slightly less than $15 / 9$, allows to frame a large lateral area, that often contains relevant information for automotive applications. The sensitivity covers both the visible and NIR spectra to allow a better object detection using a specific illumination.

A SICK LMS 211-30206 laserscanner has been placed into the front bumper. The laser has one plane, a field of view of $100^{\circ}$, minimum angular resolution of $0.25^{\circ}$, can see objects up to 80 meters, and has range and fog correction.

The camera and the laser scanner are not synchronized in hardware in order to relax the set of requirements. This means that a variable time shift exists between the samples acquired by the two sensors. This time shift is corrected via software.

NIR headlamps have been integrated in the lighting system to extend the system functionality during the night or low illumination scenario such as tunnels and parkings. Figure 3 shows the Grandeur front bumper.

Inertial data are captured through the CAN bus. The vehicle speed is obtained from the ESP sensor while two additional yaw rate sensors are installed to measure the yaw rate and the pitch rate (vertically mounted). The pitch rate is used to detect strong pitch movements that would lead to a possible wrong interpretation of the perceived data. 

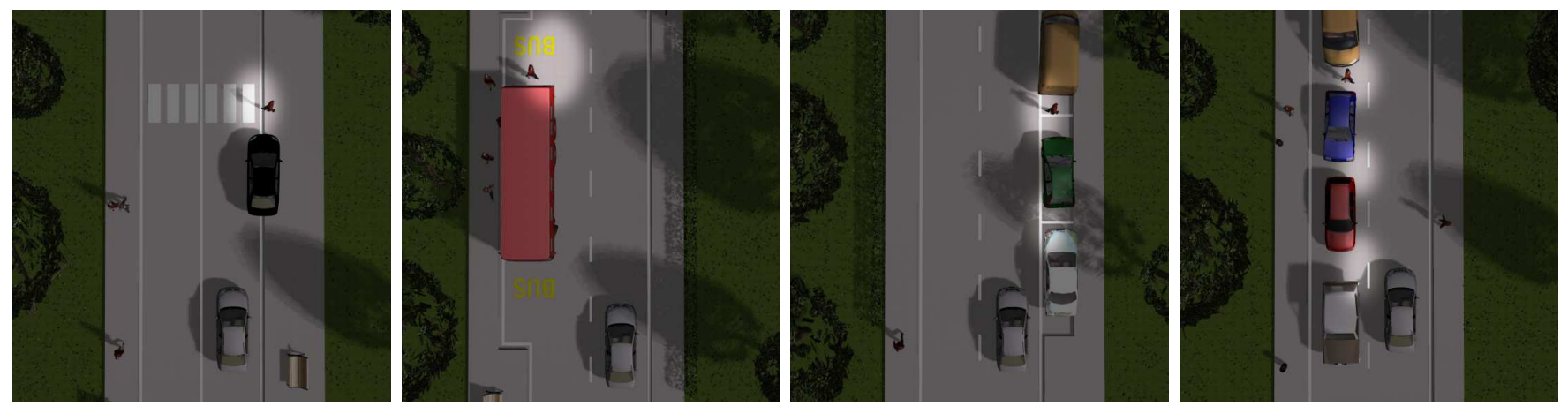

Fig. 2. Example of the scenarios considered in this work and areas of interest considered for the detection of a possible pedestrian.

The normal braking system installed on the test vehicle has been replaced with a Mando's MGH-40 ESC plus to control vehicle deceleration via CAN. The braking system incorporates a deceleration control interface capable to brake from 0 to $1.0 \mathrm{~g}$, with a resolution of $0.01 \mathrm{~g}$.

The horn was also modified so that it can be also controlled through a USB I/O board.

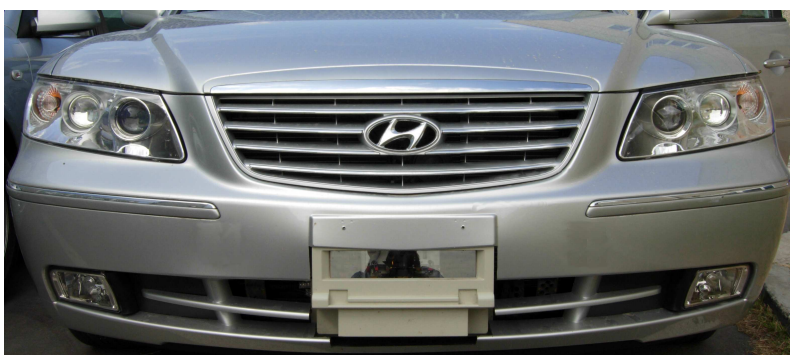

Fig. 3. A detail of the front bumper showing the laserscanner integration and the headlights.

The system designed and developed by VisLab in Parma has been replicated on a similar vehicle in Korea and is currently used by Mando to double the testing time.

\section{Functionality AND HMI}

The SDS approach described in this paper is an alternative to conventional approaches as described in the Introduction, and aims at detecting pedestrians in situations of clear danger, by limiting the search to specific areas. Besides being quicker than other systems, this method aims also at reducing the number of false detections to zero. The false positives rate is an extremely important performance index and its importance is even higher than the number of correct detections when the system is implementing non-reversible actions as a countermeasure to reduce the danger level.

The final goal of pedestrian detection systems is, in fact, to save lives and increase road safety through the use of both reversible and non-reversible systems. The system presented in this work is based on a three-level intervention strategy, the last level being a non-reversible system (automatic braking).

This strategy was conceived to give a chance to both the driver and the pedestrian to avoid the automatic braking in case they are sufficiently quick in reacting to the danger.
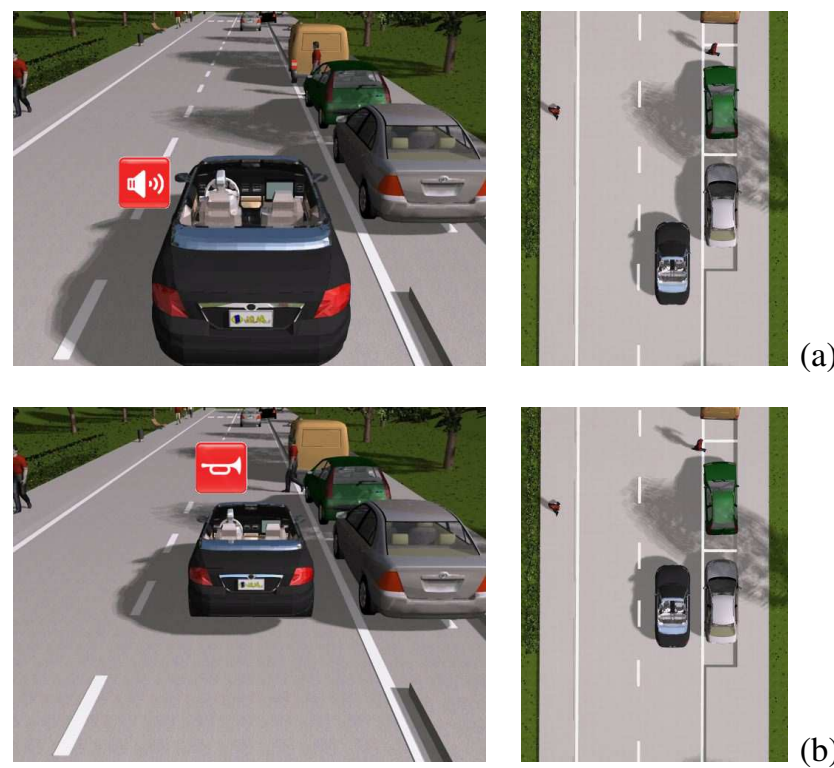

(a)
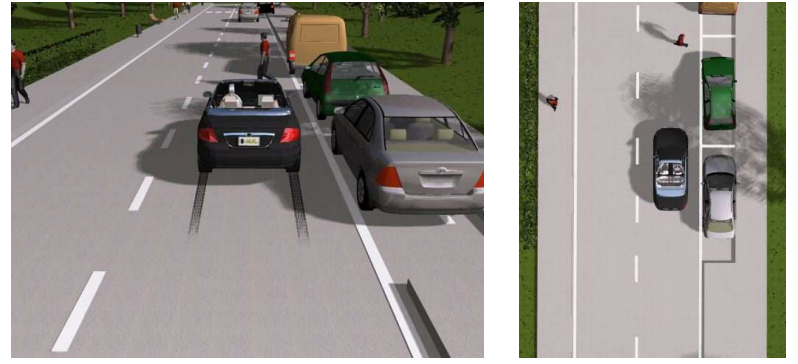

(b)

Fig. 4. The three levels of intervention: the laserscanner scans the environment and looks for obstacles that may hide pedestrians; $(a)$ when a pedestrian is spotted by vision (generally partly occluded by an obstacle), a warning is sent to the driver; $(b)$ the pedestrian, seen by vision and now confirmed by laserscanner, is tracked; if the direction of motion is towards the road center (the pedestrian is crossing the road), the horn is blown; $(c)$ when the pedestrian, detected and tracked by both sensors, enters in the deceleration area, automatic braking is triggered.

Once the pedestrian is detected with a sufficiently high confidence level, a warning is sent to the driver (figure 4.a). Shouldn't the driver react promptly to the warning, the system would issue a second level of warning by blowing the vehicle's horn (figure 4.b). This second warning is still considered a 
reversible system, although it is much more invasive than the former. The aim of this loud warning is to attract the attention of both the pedestrian itself and -once more- the driver. In case the danger level is not reduced thanks to a prompt reaction of the driver (or the pedestrian), the intelligent vehicle will trigger a non-reversible system, i.e. automatic braking (figure 4.c).

Being this a non-reversible and very invasive system, its triggering must be preceded by an extremely careful analysis of the danger level, which will have to exceed very high thresholds. Furthermore, in order to successfully field a non-reversible system, the complete processing has to be thoroughly tested against the number of false detections and will be used to assess the performance also in this work (see Section V).

\section{Algorithm}

The algorithm can be divided into two parts:

- detection of the regions of interest (ROI) using laser scanner data,

- classification of the content of these areas based on image processing.

\section{A. Focus of attention}

Dangerous areas represent ROI where the algorithm needs to search for pedestrians suddenly appearing from behind an obstacle. For the localization of these regions of interest, laser scanner data are analyzed. Laser scanner data are managed with different data structures: points, chains of segments, polylines, and finally obstacles.

Pulses provided by laser scanner need to be roto-translated, according to vehicle ego motion, in order to correct the shifts between subsequent laser scanner measurements. Data provided by ESP CAN box and by an additional yaw rate sensor are used to correct the shifts. In such a way the border of non-moving obstacles appears clearly as a single line. Pulses echoed by moving obstacles can not be reduced to a single line, since the obstacle position changes during the subsequent scans.

Points are clustered into chains of segments. Points close to the limit of the laser scanner range (about 80 meters) are joined into especially labelled segments. Isolated points are permanently discarded.

Segments of the same chain can be merged together to generate a longer segment. Considering the straight line that connects the chain start and end points, it is possible to compute the distance between this line and all the internal points of the chain: if the maximum distance is lower than a threshold the chain can be approximated by a line, otherwise it is divided into 2 lines. This method is applied iteratively until the maximum distance is lower than a fixed threshold.

Polylines obtained so far are labelled according to their shape and size; obstacles are divided into four categories:

- possible pedestrian,

- road border,

- L-shaped obstacle,

- generic obstacle.
Detected polylines are stored to build a polylines history. Previously detected polylines are roto-translated according to computed ego motion: in such a way polylines history can be used to classify polylines according to their movement. The centroid position is computed for each polyline; this information is used to compute distance from old polylines. Polylines are labeled as:

- fixed obstacle,

- moving obstacle,

- unknown obstacle.

Obstacles speed is roughly computed, as well.

Fixed obstacles are used to build the driving corridor, namely the area that may be reached by the vehicle in the near future, that corresponds to the area between fixed obstacles in which the vehicle can move.

Dangerous areas are identified by the further point of a fixed obstacle that lies in the driving corridor: these points represent the areas where a pedestrian can suddenly appear.

Furthermore laser scan data provides information on the possible presence of pedestrians in the driving corridor. Particular attention must be payed to fixed obstacles with shape similar to a pedestrian, that can be generated by obstacles larger than a pedestrian (such as vehicle) partially occluded by another obstacle: obstacles of this type are not considered as possible pedestrian but are used for dangerous areas localization. Recapping, the fixed obstacles are used to create the driving corridor and reduce the areas where possible dangerous pedestrian are searched for in the following steps. If no static object is present, possible dangerous pedestrian are searched for in the whole area in front of the vehicle.

In figure 5 the final result of the algorithm described so far is shown, more details about the algorithm are described in [1].

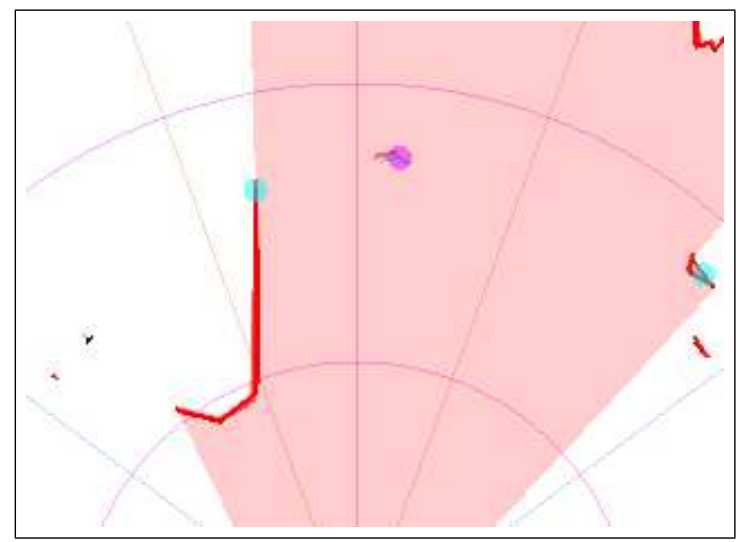

Fig. 5. Dangerous areas and possible pedestrians: Cyan circles represent dangerous areas, while violet one show a possible pedestrian.

\section{B. Areas classification}

Once the areas of attention are located, the search for pedestrians is triggered. Specific image windows are defined using a perspective mapping transformation, considering $90 \mathrm{~cm}$ 


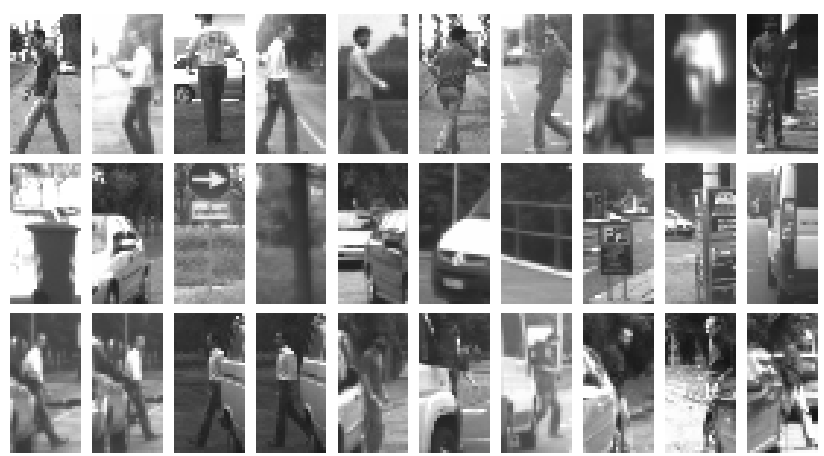

Fig. 6. Images used for training: the first row contains pedestrians, the second row contains non-pedestrians, the third row contains appearing pedestrians (i.e. partly occluded shapes).

as pedestrian width and $180 \mathrm{~cm}$ as pedestrian height; the identified areas are resampled to a fixed size $(24 \times 48$ pixels).

AdaBoost was chosen to label the regions of interest; AdaBoost is a technique widely used for the classification of pedestrians [8]. 2v, 2h, 3v, 3h, 4q Haar features were chosen for the weak classifier (see also [9], [10]).

Instead of using two classes only (pedestrians and nonpedestrians), three classes are here used:

- pedestrians,

- non-pedestrians,

- appearing pedestrians.

Appearing pedestrians are pedestrians that are initially not completely visible, i.e. partially occluded by obstacles, so only a part of the pedestrian's shape can be framed -the upper or side parts only.-

AdaBoost has been trained with about 17000 areas individuated by the previous steps of the algorithm and manually labelled (see figure 6). The images that form the training set were framed both in Italy and Korea. It becomes immediately clear that the use of public image datasets is not useful, as these images generally do not contain instances of partially occluded pedestrians.

Figure 7 shows an example of an appearing pedestrian.
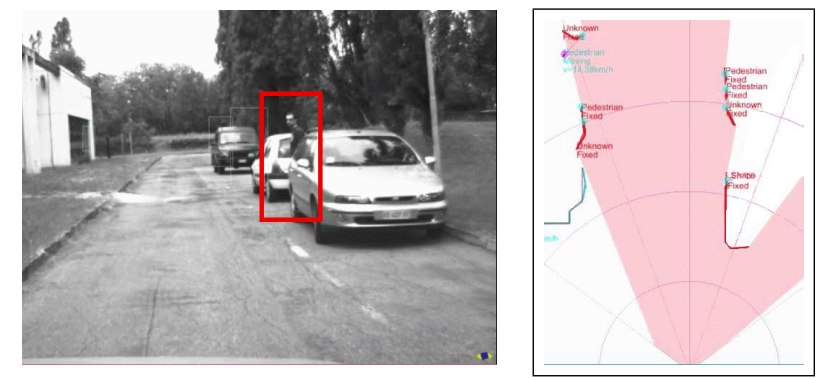

Fig. 7. Example of alert: on the left the alert identified on the image, on the right the bird-eye view. Note that the scenario is the same as in figure 4.(a)

To further increase the classification robustness another method based on shapes edges is introduced. The presence of long vertical borders that can be generated by poles or trees is checked; vertical borders symmetry is computed in order to remove particularly non-symmetrical obstacles; finally the horizontal edges histogram is considered to discard obstacles that are too short. Particular attention is payed to this last operation in order not to remove areas generated by children.

A combination of classification values of the previously described methods is used to provide the final probability of pedestrian presence. Also speed is used to classify obstacles: the probability of pedestrian presence is increased if the obstacle is moving at a reduced speed; fixed pedestrians can be detected as well, but moving pedestrians, that are the most dangerous ones, are more easily detected. This control generates positive detections also in correspondence to cyclists which, due to their nature, are indeed considered as vulnerable road users.

A simple pedestrian tracking is included in order to increase the detection rate. A pedestrian is considered as confirmed if it is tracked for a sufficiently high number of frames.

The system has been tested in night scenes as well. An additional contrast stretching is used to minimize the difference in the appearance between night-time and day-time images. It was not necessary to perform a new adaboost training with night images, as the results are satisfactory also during night tests.

\section{Warning and braking management}

A warning to the driver is triggered by the system if a pedestrian suddenly appears from behind a fixed obstacle. In the following, the actions required to perform this functionality are described.

In order to increase the probability to detect a pedestrian as soon as it becomes completely visible in front of the vehicle, the search is started even when it is still partially occluded. This search is performed within the dangerous areas detected by the laser scanner. When the content of a dangerous area is labelled as an appearing pedestrian, an internal alert is generated. The alert is triggered even if the pedestrian is not yet visible by the laser scanner (as shown in figure 7). An alert does not represent a dangerous situation per se, as the pedestrian can stay behind the obstacle without moving, thus avoiding an accident with the vehicle.

Since obstacles hiding pedestrians may have different physical structure and appearance, therefore occluding different parts of the pedestrian (a car may hide the legs, while a bus may totally occlude the visibility of the human shape), thresholds on alerts are set low. As a consequence, the number of alerts is rather high, and a confirmation is needed before the triggering of a signal to the driver. If a pedestrian appears near an alert, moving towards the center of the driving corridor, and it is tracked for a sufficiently long time, the warning signal is triggered. Although tracking introduces a delay in issuing the warning signal, it removes false positives due to obstacles erroneously classified as pedestrian and due to pedestrians suddenly stopping or changing their direction to avoid the crash.

Previous works have introduced the Region Of No Escape (RONE [11]) as the area that will be anyway occupied by 
the vehicle in the near future even if the driver steers away or brakes. Like traditional pedestrian detection systems, pedestrians present in the RONE are here detected by laser and vision fusion; the presence of a pedestrian in the RONE triggers the automatic braking, as a Collision Mitigation system (i.e. when the accident is unavoidable).

The RONE is indeed important to detect an inevitable crash, but its size and shape are defined assuming an optimal driver maneuver. Most drivers, anyway, have (i) longer reaction times and (ii) suboptimal skills; therefore an extension of the RONE is mandatory in order to act as a driving aid (avoid the crash) rather than a collision mitigation system. It has to be understood, though, that any extension of the RONE will include areas in which the vehicle has a high probability to move, but it is not $100 \%$ sure to be occupied by the vehicle in the near future (for example in case of a very skilled driver implementing an optimal avoidance maneuver).

The system presented in this work defines the deceleration area, as a suitable RONE extension designed for the specific scenario addressed by this project. In fact, pedestrians appearing from behind an obstacle might induce a reaction time longer than the time required by a driver to locate a clearly visible pedestrian. Therefore, still assuming that the driver is not distracted and has optimal driving skills, longer reaction times would enlarge the RONE. The deceleration area width is defined to be equal to vehicle width and its length proportional to vehicle speed, but anyway bounded to 40 meters, while yaw is considered constant for the trajectory computation.

Whenever a pedestrian appearing from behind an obstacle crosses the road and enters in the deceleration area, the system will activate the automatic braking, even if a skilled driver might be capable of avoiding it. This is why it is extremely important to be able to issue a warning to the driver well in advance, and provide sufficient time to perform a manual maneuver: the driver has indeed a more subtle and deeper perception of the pedestrian behavior and a manual countermeasure might be more effective than an automatic one.

In this section we introduced the concept of driving corridor, RONE, and deceleration area. It is important to note the difference between these three areas in front of the vehicle, which are used for different purposes. As shown in figure 8 the driving corridor is a jagged area edged by static obstacles present in front of the vehicle and represents the area where the vehicle can move. The deceleration area is a smoother area that represents the positions where the vehicle should move considering a fixed yaw-rate. The RONE represents the area where the vehicle will move in the near future.

As the pitch variation affects the detection, mainly generating false positives, warning and braking are disabled if a high pitch rate is measured by the sensor. Anyway generally high pitching is time limited, therefore the system is blinded for a short time interval. Warning is also disabled at very low speeds (lower than $5 \mathrm{~km} / \mathrm{h}$ ).

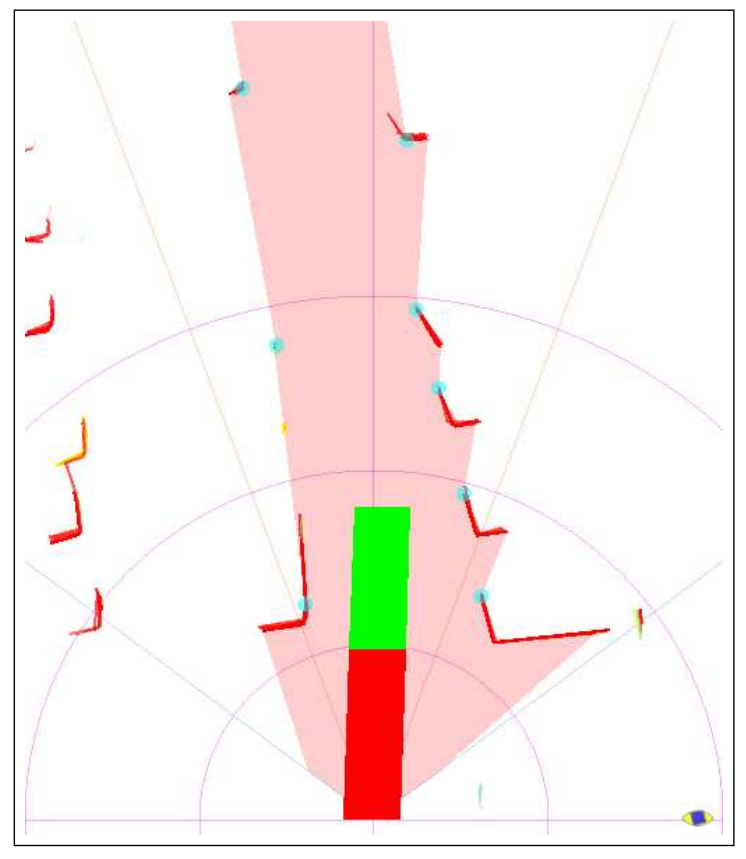

Fig. 8. Driving corridor, RONE, and deceleration area: the pink area represents the driving corridor, the red area represents the RONE, and the green area represents the deceleration area.

\section{TESTS}

To assess the system performance a large test set has been used. It contains data coming from different acquisitions for a total of about 10 hours of urban driving, including also a part of rural roads. Totally $236 \mathrm{~km}$ were driven during day and night, with different weather conditions (sunny, cloudy, rain, fog). Various scenarios are included: downtown, large and narrow roads, underground car parks, highways and rural roads. During night tests, scenarios with and without external illumination were acquired. Some specific situations were staged (such as very dangerous pedestrian crossings), but a number of dangerous scenarios were anyway framed in normal driving.

In the tests, the following performance indexes were considered:

A. number of pedestrians suddenly appearing in front of the vehicle (that must generate warnings to the driver);

B. number of pedestrians appeared in front of the vehicle that have been successfully tracked and which entered into the deceleration area (that must trigger the automatic brake);

C. number of fully visible pedestrians that entered into the deceleration area due to their own or the vehicle motion (that must trigger the automatic brake).

For each performance index the number of correct detections, false positives, and false negatives has been computed. During the tests some ambiguous situations were observed, such as a pedestrian appearing between two parked vehicles (generating the internal alert), then stopping just before crossing (the 
tracking does not issue the warning to the driver), and finally crossing the road (thus behaving like a visible pedestrian crossing the road for which no warning is planned). Such situations are considered as particular cases and will have to be analyzed separately.

A total of 24 suddenly appearing pedestrians were correctly detected in the tests (case A). Only 1 false positive is present (due to a misclassification of a parked scooter). Considering the actual camera frame rate of 15 FPS and the test duration, the false positives rate is about $2 \times 10^{-6}$ false positives per frame. Only 1 missed warning out of 11 is due to a pedestrian missdetection; the others are caused by alert miss or delayed detection: but in these cases even if the warning signal is not promptly issued to the driver, the automatic brake would have stopped the vehicle avoiding the crash if the pedestrian would have entered the deceleration area.

Regarding ambiguous cases, 4 of them generated the warning and 4 did not.

All pedestrians into the deceleration area are localized (no false negatives): 5 fall into case $B$, while 8 fall into case $\mathrm{C}$. No false positives are present, thanks to the special attention payed to develop this SDS approach. Anyway it is important to note that, for safety reasons -given that the tests were performed in real traffic, - the size of the deceleration area was increased but, nevertheless, the system behaved very satisfactorily.

Table I summarizes the results.

\begin{tabular}{|c|c|c|c|}
\hline Perform. index & Correct Detections & False Negatives & False Positives \\
\hline A & 24 & 11 & 1 \\
\hline B & 5 & 0 & 0 \\
\hline C & 8 & 0 & 0 \\
\hline
\end{tabular}

TABLE I

RESULTS OF A 10 HOURS DRIVE ( 236 KM, $\sim 540000$ IMAGES).

The analysis of the results obtained during the test also highlights good results in case of rain, when pedestrian with umbrellas were detected as well. The system is anyway not able to discriminate between multiple pedestrians moving together or in situations in which the laser scanner is not able to obtain a clear picture, for example when pedestrian hold bags or other large objects.

The total execution time is always less than $50 \mathrm{~ms}$ even in worst cases (i.e. when a high number of areas are processed) using a Core 2 Duo at $2.0 \mathrm{GHz}$ PC.

\section{CONCLUSIONS}

This paper presented a new system to increase road safety with driving aids, such as driver warning and automatic braking. This specific approach is different from traditional methods since it is specifically designed to handle a given set of scenarios and reach remarkable performance in terms of false positives in these cases.

The great advantage of an early detection of pedestrians, even when they are not enterely visible, is that a warning can be issued to the driver well in advance so that a manual maneouver can be performed. Being the driver more receptive of the intended pedestrian behavior, he/she can opt out for an avoidance maneouver that might be less invasive and dangerous than a mere braking.

The capability of localizing partly occluded pedestrians, without excessive false detections, is provided by the fusion of laser data and an innovative search called SDS.

Automatic braking is activated when the pedestrian, suddenly appeared from behind an obstacle and crossing the road, is tracked and enters in the deceleration area.

It is of paramount importance to note that the deceleration area does not represent the RONE, which -by definition- is the area that will be anyway covered by the vehicle, even if the driver steers away or brakes. Obstacles identified as pedestrians entering in the RONE will anyway trigger the automatic braking, but in this case it will act as a Collision Mitigation system (i.e. the accident is unavoidable) rather than a Driving Assistance System (i.e. braking to avoid the accident).

The next research steps will be related to consider possible enhancements in the interface model, investigating also brake modulation and release.

\section{REFERENCES}

[1] A. Broggi, P. Cerri, S. Ghidoni, P. Grisleri, and H. G. Jung, "Localization and Analysis of Critical Areas in Urban Scenarios," in Procs. IEEE Intelligent Vehicles Symposium 2008, Eindhoven, Netherlands, Jun. 2008, pp. 1074-1079.

[2] T. Gandhi and M. M. Trivedi, "Pedestrian Protection Systems: Issues, Survey, and Challenges," IEEE Trans. on Intelligent Transportation Systems, vol. 8, no. 3, pp. 413-430, Sep. 2007.

[3] M. Rapus, S. Munder, and J. D. G. Baratoff, "Pedestrian recognition using combined low-resolution depth and intensity images," in Procs. IEEE Intelligent Vehicles Symposium 2008, Eindhoven, Netherlands, Jun. 2008, pp. 632-636.

[4] K. Natroshvili, M. Schmid, M. Stephan, A. Stiegler, and T. Schamm, "Real time pedestrian detection by fusing PMD and CMOS cameras," in Procs. IEEE Intelligent Vehicles Symposium 2008, Eindhoven, Netherlands, Jun. 2008, pp. 925-929.

[5] T. Gandhi and M. Trivedi, "Image based estimation of pedestrian orientation for improving path prediction," in Procs. IEEE Intelligent Vehicles Symposium 2008, Eindhoven, Netherlands, Jun. 2008, pp. 506511.

[6] U. Franke and S. Heinrich, "Fast obstacle detection for urban traffic situations," IEEE Trans. on Intelligent Transportation Systems, vol. 03, no. 3, pp. 173-181, Sep. 2002.

[7] C. Curio, J. Edelbrunner, T. Kalinke, C. Tzomakas, and W. von Seelen, "Walking pedestrian recognition," IEEE Trans. on Intelligent Transportation Systems, vol. 01, no. 3, pp. 155-163, Sep. 2000.

[8] L. Leyrit, C. Chateau, C. Tournayre, and J.-T. Lapresté, "Association of AdaBoost and Kernel Based Machine Learning Methods for Visual Pedestrian Recognition," in Procs. IEEE Intelligent Vehicles Symposium 2008, Eindhoven, Netherlands, Jun. 2008, pp. 67-72.

[9] P. Geismann and G. Schneider, "A Two-Staged Approach to VisionBased Pedestrian Recognition Using Haar and HOG Features," in Procs. IEEE Intelligent Vehicles Symposium 2008, Eindhoven, Netherlands, Jun. 2008, pp. 554-559.

[10] P. Viola and M. Jones, "Rapid Object Detection using a Boosted Cascade of Simple Features," in Intl. Conf. on Computer Vision \& Pattern Recognition, vol. 1, Dec. 2001, pp. 511-518.

[11] K. Fuerstenberg and R. Schulz, "Laserscanners for Driver Assistance," in Procs. Intl. Workshop on Intelligent Transportation, Hamburg, Germany, Mar. 2006, pp. 155-159. 